\title{
$\nabla$ Effectiveness of Directional Lingual Exercise on Subjects with Post Stroke Dysphagia
}

\author{
IJCRR \\ Section: Healthcare \\ ISI Impact Factor \\ (2019-20): 1.628 \\ IC Value (2019): 90.81 \\ $\operatorname{SJIF}(2020)=7.893$

\section{Priyanga Seemathan*1, T Divya ${ }^{1}$, Kumaresan A², Prathap Suganthirababu²}

'Medical and Technical Sciences, Chennai-602105, India; ${ }^{2}$ Professor, Saveetha College of Physiotherapy, Saveetha Institute of Medical and Technical Sciences, Chennai-602105, India.

\section{ABSTRACT}

Objective: Dysphagia is a common swallowing problem that occurs after the stroke which has more complications. So to prevent complications, the treatment should be aimed at accelerating the recovery of swallowing function and reducing risks. Hence this study aims to evaluate the effectiveness of the directional lingual exercise on post-stroke dysphagia patients.

Methods: A quasi-experimental study was done on 30 subjects who were willing to participate. The patients were selected using a convenience sampling technique based on inclusion and exclusion criteria. The safety and simplicity of the procedure were explained to the patients and written consent was obtained from the subjects. All the subjects underwent pretest measurement that includes Functional Oral Intake Scale, water swallowing test and lingual strength and endurance measurements was taken. Followed by this the subjects were given directional lingual exercise for 10 session/day, 5 days/week for 4 weeks. The post-test evaluation was conducted as the same as in the pre-test. The values were tabulated and statistically analyzed.

Results: It was observed that there was a significant improvement in swallowing function, tongue strength and endurance $(P<0.001)$ in participants with post-stroke dysphagia.

Conclusion: From the result, it has been concluded that this intervention showed a better result for improving tongue strength and endurance. Hence this technique can be used for the rehabilitation of the subjects suffering from post-stroke dysphagia.

Key Words: Stroke, Oropharyngeal dysphagia, FOIS, Tongue strength, Tongue endurance

\section{INTRODUCTION}

Stroke is a focal neurological disturbance of presumed vascular origin that lasts for more than 24 hours which in turn produces disturbance in the brain causing motor, cognitive and sensory problems. ${ }^{1}$ Dysphagia is the common swallowing problem that occurs after the stroke which has an increased risk of death, dependency, decrease in quality of life, pneumonia occurrence, and causes prolonged stay in hospital. Swallowing difficulties often occur in post-stroke survivors in about $25 \%-62 \%{ }^{2}$

Swallowing is a voluntary physiological process that transports the ingested material and saliva from the mouth to the stomach. Stroke negatively impacts the part of the brain that controls the swallowing muscles. ${ }^{3}$ The role of the tongue is mandatory in the swallowing process as it helps to chew by pushing the food towards the teeth during grinding. ${ }^{4}$ And it also presses the crushed food against the palate and moves the bolus that initiates the swallowing process. ${ }^{5}$ The other diseases that affect the function of the tongue are tongue cancer, Beckwith-Wiedemann syndrome, an overactive thyroid, acromegaly, amyloidosis, sarcoidosis, hypothyroidism, Kawasaki disease and also in down syndrome. ${ }^{6}$

Swallowing consists of four phases that include oral preparatory, oral propulsive, pharyngeal, and oesophagal phases, in which stroke usually affects the first three phases which interrupt the voluntary control of chewing and the process of moving the food around the mouth or delaying the pharyngeal reflex. ${ }^{7}$ The tongue strength is necessary for enabling the swallowing process because the tongue is involved in the pharyngeal phase of swallowing, it produces an elevation movement and sweeps posteriorly to pass the bolus past the anterior tonsillar pillar and it stimulates the swallowing reflex. Tongue strength in a normal person is $56 \mathrm{kPa} .{ }^{8}$ And the tongue strength for post-stroke dysphagia patients reduces. ${ }^{9}$

\section{Corresponding Author:}

Priyanga Seemathan, No. 5/84, Pallavan Nagar, 3rd cross street, Madhuravoyal, Chennai-600095, India. Contact: 7598414814; Email: priyaseemathan@gmail.com

ISSN: $2231-2196$ (Print)

Received: 23.03 .2021
ISSN: 0975-5241 (Online)

Revised: 12.05 .2021
Accepted: 25.06 .2021
Published: 20.11.2021 
The tongue strength is improved using various techniques like oral motor exercise, myofunctional therapy, abilex exerciser, shaker technique, pacing and feeding strategies, postural and positional techniques, effortful swallow, Mendelsohn manoeuvre, supraglottic swallow, super-supraglottic swallow, Masako manoeuvre, head-lifting exercise, lingual isometric exercise. ${ }^{10}$

Directional lingual exercise is used to increase the tongue pressure which in turn increases the tongue strength and endurance that helps the patient to recover from swallowing difficulties. ${ }^{11}$ Thus tongue strength and endurance play an important role in swallowing function. ${ }^{12}$ Directional lingual exercise consists of forwarding, lateral and vertical task that improves the tongue strength. ${ }^{13}$ Directional lingual exercise is a particular technique that is easily understandable for the patients and it is comfortable. This does not require supervision or the help of the therapist. It can be done in the house of the patient, the hospital setting is not necessary. ${ }^{14}$

\section{MATERIAL AND METHODS}

The study was designed as a Quasi-experimental study design; the study setting was done at the Physiotherapy outpatient and inpatients department, Saveetha medical college and hospital; 30 subjects who were willing to participate were selected using convenience sampling technique based on inclusion and exclusion criteria. The inclusion criteria included both the gender under the age group of 30-50 years, the person who was positive in water swallow test, subjects with post-stroke dysphagia. The exclusion criteria included unconscious patients; people who are not willing to participate; patients with brain injury; a mouth ulcer that leads to swallowing difficulty; persons with other neurological conditions and a person with an oropharyngeal tumour. The gender and age differences are shown in Figures 1 and 2 respectively. The materials required for the study were a Device used to measure tongue strength and endurance; ice stick. The safety and simplicity of the procedure were explained to the patients and written consent was obtained from the subjects in their known language.

All the subjects underwent the pretest measurement that included FOIS, lingual strength and endurance measurements were taken. Water Swallow Test was used for selecting the patients for the study in inclusion criteria. In this test, the patient was placed in a resting position and the patient's mouth was wet with the cold water and the patient was instructed to swallow repeatedly and the number of the swallow is monitored. This test was done to involve the patients in the exercise program. The functional oral intake scale was the assessment tool used to measure the current status of the oral intake of the patient. The scale consisted of 7 levels that were mentioned based on the status of the patient. Based on the diet level and swallowing function of the patients the FOIS was measured. The strength and endurance of the tongue is measured using the device that is used to measure the tongue strength and endurance. The patient was placed in the supine lying position. The device consisted of an air-filled bulb that was placed against the roof of the mouth and instructed the patients to press the bulb with maximum pressure the patient could to measure the strength of the tongue, which was displayed in the LCD of the device and the values were noted in kilopascal. The endurance was measured by quantifying the length of the time that the patient can maintain $50 \%$ of the maximum pressure in the device. These were the pretest measured before providing the intervention. The intervention included directional lingual exercise. The training protocol included the exercise period of 10 sessions/day, 5 days/ week for 2 weeks. The patients exercised the anterior portion of the tongue using the ice stick and then with the posterior portion. The patients were asked to do the free lingual exercise in all the directions from anterior through protrusion and then to anterolateral to both the sides and then to upward and downward. After that, they were advised to do the same exercise using the ice tick for resistance. These exercises were given for 10 sessions as per the protocol. After the intervention, the Post-test evaluation was taken as the same as in the pre-test. The values were tabulated in Tables 1, 2 and 3 and statistically analyzed.

\section{STATISTICAL ANALYSIS}

Paired t-test was used to analyze significant changes between pre and post-test measurements of tongue muscle strength and endurance. Wilcoxon signed-rank test was used to analyze significant changes between pre and post-test measurements of functional oral intake scale. P-value $<0.0001$ was considered statistically significant.

\section{RESULT}

The statistical analysis made with the quantitative data table 1, 2 and 3 reveals the statistically significant difference between pre and post-test values. Figure1 and 2 show the gender statistics and the age difference in the subjects. Thus directional lingual exercise has a significant role in improving lingual strength, endurance and swallowing function.

\section{DISCUSSION}

This study was conducted to investigate the effect of directional lingual exercise on 30 subjects with post-stroke dysphagia based on the 8 -week program of isometric lingual strengthening in 10 ischemic stroke patients who showed a continuous increase in isometric lingual pressure throughout 
the protocol and increase in swallowing function. ${ }^{15}$ we included the subjects aged 30-50 years as mostly dysphagia affects aged people as done previously lingual exercise to older adults increase their isometric and swallowing pressure and used as a treatment strategy for patients with lingual weakness and swallowing disability due to other age-related conditions and also reduces dysphagia related comorbidities (pneumonia, dehydration). ${ }^{16}$

Dysphagia occurs not only in stroke it also occurs due to several other diseases such as tongue cancer, hypothyroidism, down syndrome etc., as this disease affects tongue musculature thus in turn affecting swallowing function leads to dysphagia. ${ }^{17}$ Normally tongue force is higher in males than in females. But in dysarthria caused by Amyotrophic Lateral sclerosis tongue force is reduced significantly. ${ }^{18}$ while comparing tongue strength between age groups (youngest and oldest) oldest people had the weakest tongues. ${ }^{19}$ While in case of tongue pressure comparison in gender women's were found to have significantly higher pressure than male. ${ }^{20}$ Mostly anterior tongue pressure is more than posterior and lateral pressure.

Tongue pressure remains constant across the life span. But tongue pressure reserve decreases with age. ${ }^{21}$ Old age people work hard for adequate swallowing pressure. Other age-related illnesses may put the pediatric group at high risk for dysphagia. ${ }^{22}$ we have done this directional lingual exercise protocol because it is self exercise. Patients can do it by themselves without help from the therapist. It is also easily understandable among the age group and is less cost which is in contrast with the bedside self exercise that improves both oral and pharyngeal phase and improves swallowing function. ${ }^{23}$

Our study shows that people aged between $45-50$ years are more affected than $30-45$ years. This signifies that Age plays a major role in Dysphagia. Directional lingual exercise increases the tongue strength and endurance to hold on. Tongue strengthening has a positive effect on improving oral function as well as reducing aspiration in the Pharyngeal phase as this phase is major in swallowing function. ${ }^{24}$ In this study we found the pretest and post-test values of tongue strength and tongue endurance but we fail to find which muscles are involved in swallowing. In the future individual muscle activity and strength can be clarified with EMG finding for a clear picture of the effectiveness of exercises..$^{25}$ The limitation of the study is that the muscle activity was not checked. The study can be done for patients with other neurological conditions that cause dysphagia and muscle activity can be checked.

\section{CONCLUSION}

From the result, it has been concluded that this intervention showed the better result for improving the tongue strength, endurance and swallowing function. Hence this technique can be used for the rehabilitation of the subjects suffering from post-stroke dysphagia.

\section{ACKNOWLEDGMENT}

The authors are grateful to the authorities of Saveetha College of Physiotherapy, Chennai.

\section{Conflict of Interest: Nil.}

\section{Source of Funding: Self}

\section{REFERENCES}

1. Dodds WJ, Stewart ET, Logemann JA. Physiology and radiology of the normal oral and pharyngeal phases of swallowing. AJR Am J Roentgenol. 1990 May;154(5):953-963.

2. Tibbling L, Gustafsson B. Dysphagia and its consequences in the elderly. Dysphagia. 1991;6(4):200-202.

3. Youmans SR, Stierwalt JA. Measures of tongue function related to normal swallowing. Dysphagia. 2006 Apr; 21(2):102-111.

4. Stierwalt JA, Youmans SR. Tongue measures in individuals with normal and impaired swallowing. Am J Speech Lang Pathol. 2007 May; 16(2):148-156.

5. Kim HD, Choi JB, Yoo SJ, Chang MY, Lee SW. Tongue-to-palate resistance training improves tongue strength and oropharyngeal swallowing function in subacute stroke survivors with dysphagia. J Oral Rehabil. 2017 Jan;44(1):59-64.

6. Burkhead LM, Sapienza CM, Rosenbek JC. Strength-training exercise in dysphagia rehabilitation: principles, procedures, and directions for future research. Dysphagia. 2007 Jul;22(3):251265.

7. Robbins J, Levine R, Wood J, Roecker EB, Luschei E. Age effects on lingual pressure generation as a risk factor for dysphagia. J Gerontol A Biol Sci Med Sci. 1995 Sep; 50(5):M257-262.

8. Clark HM, O'Brien K, Calleja A, Corrie SN. Effects of directional exercise on lingual strength. J Speech Lang Hear Res. 2009 Aug;52(4):1034-1047.

9. Lazarus C, Logemann JA, Huang CF, Rademaker AW. Effects of two types of tongue strengthening exercises in young normals. Folia Phoniatr Logop. 2003 Jul-Aug;55(4):199-205.

10. Robbins J, Kays SA, Gangnon RE, Hind JA, Hewitt AL, et al. The effects of lingual exercise in stroke patients with dysphagia. Arch Phys Med Rehabil. 2007 Feb;88(2):150-158.

11. Robbins J, Gangnon RE, Theis SM, Kays SA, Hewitt AL, et al. The effects of lingual exercise on swallowing in older adults. J Am Geriatr Soc. 2005 Sep;53(9):1483-1489.

12. Utanohara Y, Hayashi R, Yoshikawa M, Yoshida M, Tsuga K, et al. Standard values of maximum tongue pressure taken using newly developed disposable tongue pressure measurement device. Dysphagia. 2008 Sep;23(3):286-290.

13. Nicosia MA, Hind JA, Roecker EB, Carnes M, Doyle J, et al. Age effects on the temporal evolution of isometric and swallowing pressure. J Gerontol A Biol Sci Med Sci. 2000 Nov;55(11):M634-640.

14. McKenna VS, Zhang B, Haines MB, Kelchner LN. A Systematic Review of Isometric Lingual Strength-Training Programs in Adults With and Without Dysphagia. Am J Speech Lang Pathol. 2017 May 17;26(2):524-539. 
15. Rogus-Pulia N, Robbins J. Approaches to the rehabilitation of dysphagia in acute poststroke patients. Semin Speech Lang. 2013 Aug;34(3):154-69.

16. Ono, T., Hori, K. \& Nokubi, T. Pattern of tongue pressure on the hard palate during swallowing. Dysphagia 2004; 19:259-264.

17. Terré R, Mearin F. A randomized controlled study of neuromuscular electrical stimulation in oropharyngeal dysphagia secondary to acquired brain injury. Eur J Neurol. 2015 Apr;22(4):687644.

18. Hara K, Tohara H, Kobayashi K, Yamaguchi K, Yoshimi K, et al. Age-related declines in the swallowing muscle strength of men and women aged 20-89 years: A cross-sectional study on tongue pressure and jaw-opening force in 980 subjects. Arch Gerontol Geriatr. 2018 Sep-Oct; 78:64-70.

19. Feng X, Todd T, Lintzenich CR, Ding J, Carr JJ, et al ,. Ageingrelated geniohyoid muscle atrophy is related to aspiration status in healthy older adults. J Gerontol A Biol Sci Med Sci. 2013 Jul; 68(7):853-860.

20. Jacob P, Kahrilas PJ, Logemann JA, Shah V, Ha T. Upper oesophagal sphincter opening and modulation during swallowing. Gastroenterology. 1989 Dec;97(6):1469-1478.

Table 1: Pre and Post-test comparison of tongue muscle strength

\begin{tabular}{lcccc} 
& Mean & SD & T value & P-value \\
Pre test & 33.747 & 4.248 & 29.477 & $<0.0001$ \\
Post-test & 60.443 & 2.788 & & \\
\hline
\end{tabular}

Table 2: Pre and Post-test comparison of tongue muscle endurance

\begin{tabular}{lcccc} 
& Mean & SD & T value & P-value \\
Pre test & 6.240 & 1.011 & 28.219 & $<0.0001$ \\
Post-test & 30.203 & 4.653 & & \\
\hline
\end{tabular}

Table 3: Pre and Post-test comparison for functional oral intake scale

\begin{tabular}{|c|c|c|c|c|}
\hline $\begin{array}{l}\text { Functional oral } \\
\text { intake scale }\end{array}$ & $\mathbf{N}$ & Median & $\begin{array}{l}\mathrm{W}+\text { value } \\
\text { T value }\end{array}$ & P-value \\
\hline Pre test & 30 & 2 & $W=465.0$ & $\mathrm{P}=<0.001$ \\
\hline Post-test & & 6 & $\begin{array}{l}\mathrm{T}+=465 \\
\mathrm{~T}-=0.000 \\
\mathrm{Z} \text { value }=4.824\end{array}$ & \\
\hline
\end{tabular}

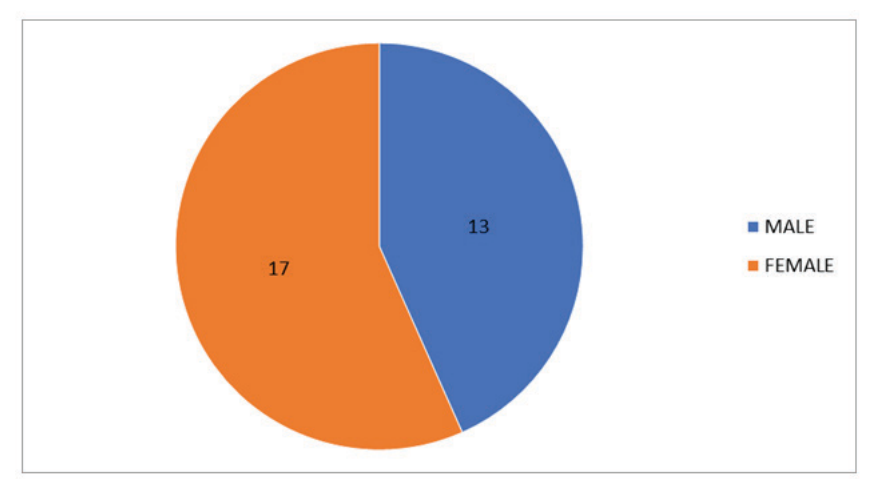

Figure 1: The graph shows that there are 13 Females and 17 Males.
21. Butler SG, Stuart A, Leng X, et al. The relationship of aspiration status with tongue and handgrip strength in healthy older adults. J Gerontol A Biol Sci Med Sci. 2011 Apr ;66(4):452-458.

22. Dworkin JP, Aronson AE, Mulder DW. Tongue force in normals and dysarthric patients with amyotrophic lateral sclerosis. J Speech Hear Res. 1980 Dec;23(4):828-37.

23. Cho YS, Oh DH, Paik YR, Lee JH, Park JS. Effects of bedside self-exercise on oropharyngeal swallowing function in stroke patients with dysphagia: a pilot study. J Phys Ther Sci. 2017;29(10):1815-1816.

24. Kim HD, Choi JB, Yoo SJ, Chang MY, Lee SW. Tongue-to-palate resistance training improves tongue strength and oropharyngeal swallowing function in subacute stroke survivors with dysphagia. J Oral Rehabil. 2017 Jan;44(1):59-64.

25. Youmans SR, Youmans GL, Stierwalt JA. Differences in tongue strength across age and gender: is there a diminished strength reserve? Dysphagia. 2009 Mar;24(1):57-65.

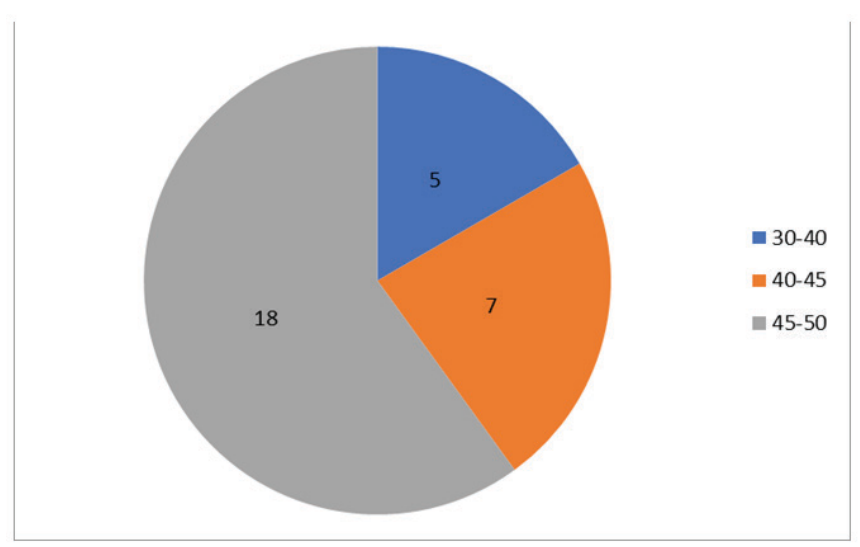

Figure 2: The graph shows the age distribution. 